CERN-TH/2000-058

hep-th/0002147

\title{
Bulk Fields in Dilatonic and Self-Tuning Flat Domain Walls
}

\author{
Donam Youm ${ }^{1}$ \\ Theory Division, CERN, CH-1211, Geneva 23, Switzerland
}

\begin{abstract}
We study the Kaluza-Klein zero modes of massless bulk fields with various spins in the background of dilatonic and self-tuning flat domain walls. We find that the zero modes of all the massless bulk fields in such domain wall backgrounds are normalizable, unlike those in the background of the non-dilatonic domain wall with infinite extra space of Randall and Sundrum. In particular, gravity in the bulk of dilatonic domain walls is effectively compactified to the Einstein gravity with vanishing cosmological constant and nonzero gravitational constant in one lower dimensions for any values of dilaton coupling parameter, provided the warp factor is chosen to decrease on both sides of the domain wall, in which case the tension of the domain wall is positive. However, unexpectedly, for the self-tuning flat domain walls, the cosmological constant of the zero mode effective gravity action in one lower dimensions does not vanish, indicating the need for additional ingredient or modification necessary in cancellation of the unexpected cosmological constant in the graviton zero mode effective action.
\end{abstract}

CERN-TH/2000-058

February, 2000

\footnotetext{
${ }^{1}$ E-mail: Donam.Youm@cern.ch
} 


\section{Introduction}

Recently, theories with extra spatial dimensions have been actively studied as solutions to the hierarchy problem in particle physics. In such scenario, all the fields of Standard model are assumed to live within the worldvolume of a brane, whereas gravity can freely propagate in the extra space as well as within the worldvolume of the brane. Although the earlier proposal $[1,2]$ attempts to solve the hierarchy problem by assuming large enough extra space, the hierarchy problem is recast into the problem of large ratio between the (fundamental) TeV Planck scale and the compactification scale of the extra spatial dimensions. Later proposal $[3,4,5]$ by Randall and Sundrum (RS) relies on the exponentially decreasing warp factor in the metric of the non-factorizable spacetime for solving the hierarchy problem. The RS model does not require large enough extra dimensions for solving the hierarchy problem; it is the decreasing warp factor that accounts for much smaller electroweak scale compared to the Planck scale. Another novelty of the RS model is that the extra dimensions can even be infinite in size because gravity in the bulk of the RS domain wall is effectively localized [4] around the domain wall.

In our previous works $[6,7]$, we showed that the RS type scenario can be extended to the dilatonic domain walls. In fact, one is bound to consider dilatonic domain walls if one wishes to embed the RS type scenarios within string theories, since most of the five-dimensional domain walls in string theories are dilatonic. We showed that the warp factor decreases [6] (and becomes zero at finite distance away from the wall) and the gravity in the bulk of the dilatonic domain walls is effectively compactified to the Einstein gravity with vanishing cosmological constant and nonzero gravitational constant in one lower dimensions [7], if the cosmological constant is negative $(\Lambda=$ $-2 Q^{2} / \Delta>0$, therefore $\Delta<0$, in our convention for the action). In this paper, we show that even for the positive cosmological constant $(\Lambda<0$ in our convention and therefore $\Delta>0$ ) the bulk gravity is effectively compactified to the Einstein gravity with vanishing cosmological constant and nonzero gravitational constant in one lower dimensions, provided the warp factor is chosen to decrease on both sides of the wall and the extra spatial dimension is cut off through the introduction of another domain wall. Note, for any values of the dilaton coupling parameter (therefore, for any values of $\Delta$ ), the warp factor can be chosen to be increasing or decreasing within the finite allowed extra spatial coordinate interval around the wall. (In our previous works, we considered the case when the warp factor decreases [increases] for $\Delta<0[\Delta>0]$.) Therefore, the RS type scenario can be realized for dilatonic domain walls with any values of the dilaton coupling parameter, so for any dilatonic domain walls in string theories.

The RS scenario relies on the fine-tuning of the domain wall tension, whose value is 
determined by the five-dimensional cosmological constant. It is pointed out [8] that the fine-tuned value of the domain wall tension is required by supersymmetry. However, so far it has been observed $[8,9,10,11,12,13,14]$ that supersymmetry rather requires increasing warp factor at least on one side of the wall, instead of decreasing warp factor on both sides. Furthermore, it is not guaranteed that the fine-tuned value of the domain wall tension does not receive quantum corrections after the SUSY breaking. Recently, new type of domain wall solutions which do not suffer from such problem were constructed $[15,16]$. These solutions, called "self-tuning flat domain wall", are obtained within the model with bulk dilaton but without bulk cosmological constant term. Novelty of such domain wall solutions is that a static domain wall solution with the Poincaré invariance in one lower dimensions exists for any values of the domain wall tension. So, even if the quantum effect corrects the domain wall tension, the Poincaré invariance is not disturbed. With a choice of the warp factor that has singularity at finite distance away from the wall on both sides, one therefore would expect that such domain wall effectively compactifies the five-dimensional gravity to four-dimensional gravity with vanishing cosmological constant regardless of the quantum corrections on the domain wall tension.

It is the purpose of this paper to study the Kaluza-Klein (KK) zero modes of the massless fields with various spins in the bulk of the dilatonic and the self-tuning flat domain walls. (The previous works on bulk fields in the non-dilatonic domain wall can be found for example in Refs. [17, 18, 19, 20, 21, 22, 23, 24].) Although the brane world scenarios assume that only gravity lives in the bulk and the remaining fields are confined within the brane worldvolume, it would be interesting to study various fields in the bulk. One of the reasons is that if we want to embed the brane world scenarios within string theories, we have to consider bulk fields compactified from ten or eleven dimensions, unless we want to truncate them ad hoc just for the purpose of letting only gravity (and the dilaton for the dilatonic domain wall case) live in the bulk. We find that the KK zero modes of massless fields of various spins in the bulk of the dilatonic and the self-tuning flat domain walls are normalizable, whereas the KK zero modes of only the massless spin-0 and spin-2 fields in the bulk of non-dilatonic RS domain wall with infinite extra spatial dimensions $[4,5]$ are normalizable. In general, we find that the KK zero modes for the integer spin bulk fields are independent of the extra spatial coordinate, whereas those of the half-integer spin fields depend on it. An unexpected result is that, contrary to the claims in Refs. [15, 16], the KK zero mode effective action (in one lower dimensions) for the gravity in the bulk of the selftuning flat domain wall has non-vanishing cosmological constant term. This seems to be an indication of the need for additional yet unknown ingredient in the picture of the self-tuning flat domain wall necessary in cancellation of the unexpected cosmological constant term in the effective action. 
The paper is organized as follows. In section 2, we discuss the dilatonic and the self-tuning flat domain walls. We reparametrize the dilatonic domain walls, which we previously studied, in terms of the bulk cosmological constant. We rederive the selftuning flat domain walls with different parametrization (from the one in Ref. [16]) which we find more convenient. In section 3, we obtain the KK zero modes for the bulk fields with various spins and their effective actions in one lower dimensions.

\section{Domain Wall Solutions}

In this section, we discuss the dilatonic domain walls that we studied previously and the self-tuning flat domain wall solutions that are constructed in Refs. [15, 16]. Although detailed derivation is given in Ref. [16], we rederive the self-tuning flat domain wall solutions because we wish to use different parametrization of the solutions, which we find more convenient. Also, even if only five-dimensional domain walls are phenomenologically of interest, we derive the domain wall solutions in arbitrary dimensions just for the purpose of generality and because such solutions might be useful for other studies.

Generally, the total action is the sum of the $D$-dimensional action $S_{\text {bulk }}$ in the bulk of the domain wall and the $(D-1)$-dimensional action $S_{\mathrm{DW}}$ on the domain wall worldvolume:

$$
S=S_{\mathrm{bulk}}+S_{\mathrm{DW}}
$$

For the domain wall solutions under consideration in the paper, the $D$-dimensional action contains the bulk action for the domain wall solutions:

$$
S_{\text {bulk }} \supset \frac{1}{2 \kappa_{D}^{2}} \int d^{D} x \sqrt{-G}\left[\mathcal{R}_{G}-\frac{4}{D-2} \partial_{M} \phi \partial^{M} \phi+e^{-2 a \phi} \Lambda\right],
$$

and the $(D-1)$-dimensional action contains the following worldvolume action for the domain wall solutions:

$$
S_{\mathrm{DW}} \supset-\int d^{D-1} x \sqrt{-\gamma} f(\phi)
$$

where $\gamma$ is the determinant of the induced metric $\gamma_{\mu \nu}=\partial_{\mu} X^{M} \partial_{\nu} X^{N} G_{M N}$ on the domain wall worldvolume, $M, N=0,1, \ldots, D-1$ and $\mu, \nu=0,1, \ldots, D-2$. Note, for the purpose of following the notation of our previous works $[6,7]$, we are using the different convention for the sign of the cosmological constant $\Lambda$ and the sign and the value of the dilaton coupling parameter $a$ from that in Ref. [16]. So, in our case, the positive [the negative] $\Lambda$ (with $a=0$ ) corresponds to the AdS space [dS space]. We are interested in finding solutions with the Poincaré invariance in $(D-1)$ dimensions. The general Ansätze for the fields with such symmetry are

$$
G_{M N} d x^{M} d x^{N}=e^{2 A(y)}\left[-d t^{2}+d x_{1}^{2}+\cdots+d x_{D-2}^{2}\right]+d y^{2}
$$


and $\phi=\phi(y)$.

The $\Lambda \neq 0 \neq a$ case, considered in Ref. [16], is nothing but the extreme dilatonic domain wall solutions studied in our previous works $[6,7]$. In this case, $f(\phi)$ in the worldvolume action has to take a specific form $f(\phi)=\sigma_{D W} e^{-a \phi}$ with the energy density $\sigma_{D W}$ of the domain walls taking the fine-tuned value determined by the bulk cosmological constant $\Lambda$ and the dilaton coupling parameter $a$. It is observed in Ref. [6] that the extra spatial coordinate $y$ terminates at finite nonzero value due to the singularity. It is explicitly shown in Ref. [7] that the gravity in the bulk of the dilatonic domain wall is effectively compactified to the Einstein gravity in one lower dimensions with nonzero gravitational constant and vanishing cosmological constant. The solution, reparametrized in terms of the bulk cosmological constant $\Lambda$ and the dilaton coupling parameter $a$, has the following form:

$$
e^{2 A}=(K y+1)^{\frac{8}{(D-2)^{2} a^{2}}}, \quad \phi=\frac{1}{a} \ln (K y+1)+C,
$$

where $C$ is an integration constant and

$$
K= \pm \frac{(D-2) a^{2}}{2} e^{-a C} \sqrt{\frac{D-2}{4(D-1)-a^{2}(D-2)^{2}} \Lambda}
$$

The requirement of the term inside the square root in Eq. (6) to be positive fixes the sign of the cosmological constant $\Lambda$ to be positive [negative] for $a^{2}<4(D-1) /(D-2)^{2}$ $\left[a^{2}>4(D-1) /(D-2)^{2}\right]$. This is in accordance with the expression $\Lambda=-2 Q^{2} / \Delta$, where $\Delta \equiv(D-2) a^{2} / 2-2(D-1) /(D-2)$, that we used in our previous works $[6,7]$. The sign \pm in Eq. (6) has to be chosen such that the spacetime metric (4) with the warp factor in Eq. (5) has a singularity at finite $y$, if we want the gravity in the bulk of the domain wall to be effectively compactified. So, we choose the plus [minus] sign for the region $y<0[y>0]$. With this choice of the signs, the energy density of the wall, determined by solving the boundary condition at $y=0$, takes the following positive value:

$$
\sigma_{\text {DW }}=\frac{4}{\kappa_{D}^{2}} \sqrt{\frac{D-2}{4(D-1)-a^{2}(D-2)^{2}} \Lambda} .
$$

With a choice of the same signs on both sides of the wall, $\sigma_{D W}=0$. With a choice such that there is no singularity at $y \neq 0, \sigma_{D W}$ is negative with the same absolute value as Eq. (7). These properties of the extreme dilatonic domain wall are essentially what we discussed in our previous works [6, 7], except the choice of the sign \pm in Eq. (6). In our previous works, we considered the case when the metric has the singularity at finite $y$ for $\Delta<0$, but no singularity at finite $y$ for $\Delta>0$. However, we see from the above that there is another choice of signs which gives the opposite singularity properties. So, the correct statement has to be that for any values of the dilaton coupling parameter a, one can choose the warp factor to be decreasing on both sides of the wall by choosing 
the sign \pm in Eq. (6) such that $K<0[K>0]$ for $y>0[y<0]$, in which case $\sigma_{\mathrm{DW}}>0$ and the there are singularities on both sides of the wall. However, we note that for the $\Delta>0$ case (or the $a^{2}>4(D-1) /(D-2)^{2}$ case), the cosmological constant $\Lambda$ has the opposite sign, i.e., the bulk spacetime is dS-like.

Now, we consider the $\Lambda=0$ case. In deriving the solutions, we choose the static gauge for the domain wall worldvolume action, so $\gamma_{\mu \nu}=\delta_{\mu}^{M} \delta_{\nu}^{N} G_{M N}$. With the $(D-1)$ dimensional Poincaré invariant Ansätze for the fields, the equation of motion for the dilaton $\phi$ takes the following form:

$$
\frac{4}{D-2} \frac{1}{\kappa_{D}^{2}}\left[(D-1) A^{\prime} \phi^{\prime}+\phi^{\prime \prime}\right]=f^{\prime}(\phi) \delta(y),
$$

and the Einstein's equations can be brought to the following forms:

$$
\begin{gathered}
\frac{1}{2}(D-2)(D-1)\left(A^{\prime}\right)^{2}-\frac{2}{D-2}\left(\phi^{\prime}\right)^{2}=0, \\
\frac{1}{\kappa_{D}^{2}}\left[(D-2) A^{\prime \prime}+\frac{4}{D-2}\left(\phi^{\prime}\right)^{2}\right]+f(\phi) \delta(y)=0,
\end{gathered}
$$

where the primes on $\phi$ and $A$ mean differentiations with respect to $y$ and the prime on $f(\phi)$ means differentiation with respect to $\phi$. Eq. (9) implies the following relation between $\phi$ and $A$ :

$$
A^{\prime}=\eta \frac{2}{D-2} \frac{1}{\sqrt{D-1}} \phi^{\prime} \quad(\eta \equiv \pm 1)
$$

So, Eqs. (8) and (10) can be brought to the following forms:

$$
\begin{gathered}
\frac{4}{D-2} \frac{1}{\kappa_{D}^{2}}\left[\eta \frac{2}{D-2} \sqrt{D-1}\left(\phi^{\prime}\right)^{2}+\phi^{\prime \prime}\right]=f^{\prime}(\phi) \delta(y), \\
\frac{1}{\kappa_{D}^{2}}\left[\eta \frac{2}{\sqrt{D-1}} \phi^{\prime \prime}+\frac{4}{D-2}\left(\phi^{\prime}\right)^{2}\right]=-f(\phi) \delta(y) .
\end{gathered}
$$

In the bulk $(y \neq 0)$, Eqs. (12) and (13) take the same form and one can solve one of them to obtain the general solution for $\phi$. In Ref. [16], the solution is parametrized in the following way:

$$
\phi=\eta \frac{D-2}{2} \frac{1}{\sqrt{D-1}} \ln \left|\frac{2}{D-2} \sqrt{D-1} y+c\right|+d
$$

but we find it more convenient to parametrize the solution in the following way:

$$
\phi=\eta \frac{D-2}{2} \frac{1}{\sqrt{D-1}} \ln (K y+1)+C .
$$


Then, from Eq. (11) we obtain the following standard form of the metric warp factor:

$$
e^{2 A}=(K y+1)^{\frac{2}{D-1}}
$$

Note, Ref. [16] considers the possibility of having an arbitrary constant factor in front of this warp factor, which results from the integration constant in integrating Eq. (11) with respect to $y$ as well as from the integration constant $C$ in Eq. (15). However, such constant factor can be absorbed by rescaling the $(D-1)$-dimensional coordinates $x^{\mu}$ in the metric. The only effect of such constant factor on the KK zero modes of massless bulk fields is values of their normalization constants.

Note, $\eta$ 's in the above equations and the dilaton solution can take any (independent) signs on each side of the domain wall. So, we have two sets of equations (12) and (13), one for each side, with $\eta$ replaced by $\eta_{+}\left[\eta_{-}\right]$for the set of equations in the region $y>0$ $[y<0]$. And we have the following expressions for the warp factor:

$$
e^{2 A}=\left\{\begin{array}{ll}
\left(K_{+} y+1\right)^{\frac{2}{D-1}}, & y>0 \\
\left(K_{-} y+1\right)^{\frac{2}{D-1}}, & y<0
\end{array},\right.
$$

and the dilaton

$$
\phi=\left\{\begin{array}{ll}
\eta_{+} \frac{D-2}{2} \frac{1}{\sqrt{D-1}} \ln \left(K_{+} y+1\right)+C, & y>0 \\
\eta_{-} \frac{D-2}{2} \frac{1}{\sqrt{D-1}} \ln \left(K_{-} y+1\right)+C, & y<0
\end{array},\right.
$$

where we have imposed the continuity of $\phi$ at $y=0$, i.e., $\phi\left(0^{+}\right)=\phi\left(0^{-}\right)$. In the following, we impose the boundary conditions at $y=0$ on the general solutions (17) and (18) to determine the integration constants $K_{ \pm}$in terms of the parameters in the actions (2) and (3) and the other integration constant.

First, we consider the case when $\eta$ 's in the above equations and the solutions on the two sides of the domain wall have the opposite signs. Namely, we choose $\eta_{+}=-\eta_{-}=\eta$, where $\eta= \pm 1$. By imposing the boundary conditions on $\phi$ at $y=0$, we obtain the following expressions for $K_{ \pm}$:

$$
\begin{aligned}
& K_{+}=\frac{1}{2} \kappa_{D}^{2}\left[\eta \frac{\sqrt{D-1}}{2} f^{\prime}(C)-\frac{D-1}{D-2} f(C)\right] \\
& K_{-}=\frac{1}{2} \kappa_{D}^{2}\left[\eta \frac{\sqrt{D-1}}{2} f^{\prime}(C)+\frac{D-1}{D-2} f(C)\right]
\end{aligned}
$$

With a choice $f(\phi)=\sigma_{\mathrm{DW}} e^{b \phi}, K_{ \pm}$take the following forms:

$$
\begin{aligned}
& K_{+}=\frac{1}{2} \kappa_{D}^{2} \sigma_{\mathrm{DW}} e^{b C}\left[\eta \frac{\sqrt{D-1}}{2} b-\frac{D-1}{D-2}\right], \\
& K_{-}=\frac{1}{2} \kappa_{D}^{2} \sigma_{\mathrm{DW}} e^{b C}\left[\eta \frac{\sqrt{D-1}}{2} b+\frac{D-1}{D-2}\right] .
\end{aligned}
$$


From these expressions for $K_{ \pm}$, we see that a non-trivial solution does not exist when $b= \pm 2 \sqrt{D-1} /(D-2)$. As we will see in the following, non-trivial solutions for this case exist when $\eta$ 's on the two sides of the wall have the same signs.

Second, we consider the case when $\eta$ 's on the two sides of the domain wall have the same signs. Namely, we choose $\eta_{+}=\eta_{-}=\eta$, where $\eta= \pm 1$. By imposing the boundary conditions on $\phi$ at $y=0$, we obtain the following relation between $K_{+}$and $K_{-}$:

$$
K_{+}-K_{-}=\eta \frac{\sqrt{D-1}}{2} \kappa_{D}^{2} f^{\prime}(C)=-\frac{D-1}{D-2} \kappa_{D}^{2} f(C) .
$$

With a choice $f(\phi)=\sigma_{\mathrm{DW}} e^{b \phi}$, the relation becomes:

$$
K_{+}-K_{-}=\eta \frac{\sqrt{D-1}}{2} \kappa_{D}^{2} b \sigma_{\mathrm{DW}} e^{b C}=-\frac{D-1}{D-2} \kappa_{D}^{2} \sigma_{\mathrm{DW}} e^{b C},
$$

from which we see that $b$ is fixed to take the following values:

$$
b=-2 \eta \frac{\sqrt{D-1}}{D-2} .
$$

Note, we have freedom of choosing any values of $K_{ \pm}$as long as the constraint (21) or (22) is satisfied.

We now comment on novelty $[15,16]$ of the domain wall solution with $\Lambda=0$. First of all, as we can see from the explicit expressions for $K_{ \pm}$, the $(D-1)$-dimensional Poincaré invariant solution exists for any values of the energy density $\sigma_{D W}$ of the domain wall (and the parameter $b$ ). (In fact, the independent free parameters ${ }^{2}$, which can take any values, of the $\Lambda=0$ solutions are the parameters of $f(\phi)$, i.e., $\sigma_{D W}$ and $b$ for the $f(\phi)=\sigma_{D W} e^{b \phi}$ case, and $C$.) So, $\sigma_{D W}$ (and $b$ ) needs not be fine-tuned and the quantum correction on $\sigma_{D W}$ does not disturb the $(D-1)$-dimensional Poincaré invariance. Second, in general $K_{+} \neq-K_{-}$, namely the solution has no $\mathbf{Z}_{2}$ invariance under $y \rightarrow-y$. The $\mathbf{Z}_{2}$ invariance is not possible for the $\eta_{+}=-\eta_{-}$case, but one can choose the values of $K_{ \pm}$such that the $\mathbf{Z}_{2}$ invariance is achieved for the $\eta_{+}=\eta_{-}$ case. So, the $\mathbf{Z}_{2}$ invariant domain wall solution obtained in Ref. [15] is a special case of the self-tuning flat domain wall solution with $\eta_{+}=\eta_{-}$constructed in Ref. [16]. All of these special features of the self-tuning flat domain wall solutions are manifestly recognizable, if we use our parametrization (15) of the solutions, instead of the one (14) used in Ref. [16]. Also, the solutions take simple and attractive forms with our parametrization.

\footnotetext{
${ }^{2}$ Another free parameter, namely the integration constant which results from integrating Eq. (11) with respect to $y$, can be removed by rescaling the $(D-1)$-dimensional coordinates $x^{\mu}$, as we mentioned previously.
} 


\section{The Kaluza-Klein Zero Modes of Bulk Fields}

In this section, we study the KK zero modes of massless fields in the bulk of various domain walls discussed in the previous section. Although we are interested in the KK zero modes of massless bulk fields, we shall first obtain general equations satisfied by any KK modes of massive (for the spin-0 and spin-1 cases) bulk fields, and then we shall restrict ourselves to the special case of the KK zero modes of massless bulk fields. In this section, we consider the five-dimensional domain walls, only, since only these are phenomenologically of interest. So, in the following we rewrite the explicit domain wall solutions specifically for the $D=5$ case:

- dilatonic domain wall:

The warp factor and the dilaton for the domain wall with singularities at finite $y$ (therefore, decreasing warp factor) on both sides of the wall are given by

$$
e^{2 A}=(1-K|y|)^{\frac{8}{9 a^{2}}}, \quad \phi=\frac{1}{a} \ln (1-K|y|)+C
$$

where the parameter $K$ and the tension $\sigma_{\mathrm{DW}}$ of the wall take the following fixed values determined by the bulk cosmological constant $\Lambda$ :

$$
K=\frac{3}{2} a^{2} e^{-a C} \sqrt{\frac{3}{16-9 a^{2}} \Lambda}, \quad \sigma_{\mathrm{DW}}=\frac{4}{\kappa_{5}^{2}} \sqrt{\frac{3}{16-9 a^{2}} \Lambda}
$$

- self-tuning flat domain wall:

The warp factor and the dilaton are given by

$$
\begin{aligned}
e^{2 A} & =\left\{\begin{array}{ll}
\left(K_{+} y+1\right)^{\frac{1}{2}}, & y>0 \\
\left(K_{-} y+1\right)^{\frac{1}{2}}, & y<0
\end{array},\right. \\
\phi & =\left\{\begin{array}{ll}
\eta_{+} \frac{3}{4} \ln \left(K_{+} y+1\right)+C, & y>0 \\
\eta_{-} \frac{3}{4} \ln \left(K_{-} y+1\right)+C, & y<0
\end{array} .\right.
\end{aligned}
$$

First, for the $\eta_{+}=-\eta_{-}=\eta$ case, the parameters $K_{ \pm}$are given in general by

$$
\begin{aligned}
& K_{+}=\frac{1}{2} \kappa_{5}^{2}\left[\eta f^{\prime}(C)-\frac{4}{3} f(C)\right] \\
& K_{-}=\frac{1}{2} \kappa_{5}^{2}\left[\eta f^{\prime}(C)+\frac{4}{3} f(C)\right] .
\end{aligned}
$$

With a choice $f(\phi)=\sigma_{\mathrm{DW}} e^{b \phi}$,

$$
\begin{aligned}
& K_{+}=\frac{1}{2} \kappa_{5}^{2} \sigma_{\mathrm{DW}} e^{b C}\left[\eta b-\frac{4}{3}\right], \\
& K_{-}=\frac{1}{2} \kappa_{5}^{2} \sigma_{\mathrm{DW}} e^{b C}\left[\eta b+\frac{4}{3}\right] .
\end{aligned}
$$


Second, for the $\eta_{+}=\eta_{-}=\eta$ case, the parameters $K_{ \pm}$are in general constrained to satisfy

$$
K_{+}-K_{-}=\eta \kappa_{5}^{2} f^{\prime}(C)=-\frac{4}{3} \kappa_{5}^{2} f(C) .
$$

With a choice $f(\phi)=\sigma_{\mathrm{DW}} e^{b \phi}$,

$$
K_{+}-K_{-}=\eta \kappa_{5}^{2} b \sigma_{\mathrm{DW}} e^{b C}=-\frac{4}{3} \kappa_{5}^{2} \sigma_{\mathrm{DW}} e^{b C} .
$$

\subsection{Scalar field}

The action for the bulk scalar field $\Phi\left(x^{\mu}, y\right)$ is

$$
S_{\text {bulk }} \supset \frac{1}{2} \int d^{4} x d y \sqrt{-G}\left[G^{M N} \partial_{M} \Phi \partial_{N} \Phi-m^{2} \Phi^{2}\right]
$$

where $G_{M N}$ is the metric (4) for the domain wall solution. From this action, we obtain the following the equation of motion for the scalar:

$$
\partial_{M}\left[\sqrt{-G} G^{M N} \partial_{N} \Phi\right]+\sqrt{-G} m^{2} \Phi=0
$$

which takes the following form after the metric (4) is substituted:

$$
e^{2 A} \eta^{\mu \nu} \partial_{\mu} \partial_{\nu} \Phi+\partial_{y}\left[e^{4 A} \partial_{y} \Phi\right]+m^{2} e^{4 A} \Phi=0
$$

To consider the KK mode of $\Phi$ with mass $m_{n}$, only, we decompose $\Phi$ as

$$
\Phi\left(x^{\mu}, y\right)=\varphi_{n}\left(x^{\mu}\right) f_{n}(y)
$$

and require $\varphi_{n}\left(x^{\mu}\right)$ to satisfy the following Klein-Gordon equation for a scalar with mass $m_{n}$ in flat four-dimensional spacetime:

$$
\left[\eta^{\mu \nu} \partial_{\mu} \partial_{\nu}+m_{n}^{2}\right] \varphi_{n}=0
$$

Then, the equation of motion (33) for $\Phi$ reduces to the following form of Sturm-Liouville equation:

$$
\partial_{y}\left[e^{4 A} \partial_{y} f_{n}\right]+m^{2} e^{4 A} f_{n}=m_{n}^{2} e^{2 A} f_{n}
$$

The operator $\mathcal{L}=\partial_{y}\left(e^{4 A} \partial_{y}\right)+m^{2} e^{4 A}$ is self-adjoint, provided the boundary condition $\left.f_{n}^{*} e^{4 A} f_{m}^{\prime}\right|_{y=a} ^{y=b}=0$ is satisfied, where $a \leq y \leq b$ is the interval in which the domain wall metric (4) is well-defined. In this case, the eigenvalues $m_{n}^{2}$ are real and the eigenfunctions $f_{n}$ with different eigenvalues are orthogonal to each other with respect to the weighting function $w(y)=e^{2 A}$, i.e., $\int_{b}^{a} d y f_{m}^{*}(y) f_{n}(y) w(y)=0$ for $m_{m}^{2} \neq m_{n}^{2}$. 
In term of a new $y$-dependent function $\tilde{f}_{n}=e^{2 A} f_{n}$, Eq. (36) takes the following form of the Schrödinger equation:

$$
-\frac{d^{2} \tilde{f}_{n}}{d y^{2}}+V(y) \tilde{f}_{n}=m^{2} \tilde{f}_{n}
$$

with the potential

$$
V(y)=2\left[A^{\prime \prime}+2\left(A^{\prime}\right)^{2}+\frac{1}{2} m_{n}^{2} e^{-2 A}\right] .
$$

From now on, we shall be interested in only the zero mode $\left(m_{0}=0\right)$ of the massless bulk scalar $(m=0)$.

First, we consider the dilatonic domain wall solution (24). The potential (38) in the Schrödinger equation (37) takes the following form:

$$
V(y)=\frac{8 K^{2}}{81 a^{4}} \frac{8-9 a^{2}}{(1-K|y|)^{2}}-\frac{16 K}{9 a^{2}} \delta(y) .
$$

The $\mathbf{Z}_{2}$ invariant solution to the Schrödinger equation that satisfies the boundary condition $\tilde{f}_{0}^{\prime}\left(0^{+}\right)-\tilde{f}_{0}^{\prime}\left(0^{-}\right)=-\frac{16 K}{9 a^{2}} \tilde{f}_{0}(0)$ has the form $\tilde{f}_{0}(y) \sim(1-K|y|)^{\frac{8}{9 a^{2}}}$. So, the KK zero mode is constant: $f_{0}(y)=e^{-2 A} \tilde{f}_{0}(y)=$ constant. This constant zero mode is normalizable ${ }^{3}$ with respect to the weighting function $w(y)=e^{2 A}$. The normalization constant is $N_{0}=\sqrt{\left(\frac{1}{2}+\frac{4}{9 a^{2}}\right) K}$, i.e., $\int_{-1 / K}^{1 / K} d y f_{0}^{*}(y) f_{0}(y) w(y)=1$ with $f_{0}(y)=N_{0}$ and $w(y)=e^{2 A}$. Indeed, by substituting the KK zero-mode $\Phi=\varphi_{0} f_{0}$ into the action (31) for the massless bulk scalar $(m=0)$, we obtain the following action for the massless scalar in the flat four-dimensional spacetime:

$$
\begin{aligned}
\frac{1}{2} \int d^{4} x d y \sqrt{-G} G^{M N} \partial_{M} \Phi \partial_{N} \Phi & =\frac{1}{2} \int_{-1 / K}^{1 / K} d y e^{2 A} f_{0}^{2} \int d x^{4} \eta^{\mu \nu} \partial_{\mu} \varphi_{0} \partial_{\nu} \varphi_{0} \\
& =\frac{1}{2} \int d x^{4} \eta^{\mu \nu} \partial_{\mu} \varphi_{0} \partial_{\nu} \varphi_{0}
\end{aligned}
$$

As we pointed out in the previous section, had we chosen to have a constant factor (due to the integration constants) in the warp factor $e^{2 A}$, the normalization constant $N_{0}$ would have had dependence on the constant factor (as can be seen from the normalization relation $\int d y f_{0}^{2} e^{2 A}=1$ ), but the effective action (40) for the KK zero mode in one lower dimensions does not depend on the constant factor. This generally holds for the case of the self-tuning flat domain walls and for other bulk fields to be discussed in the following subsections.

\footnotetext{
${ }^{3}$ In the case of the non-dilatonic RS domain wall, the KK zero mode $f_{0}=$ constant is also normalizable: $\int_{-\infty}^{\infty} d y f_{0}^{2} e^{2 A} \sim \int_{-\infty}^{\infty} d y e^{-2 k|y|}<\infty$.
} 
Second, for the self-tuning flat domain wall solution (26), the potential takes the following form ${ }^{4}$ :

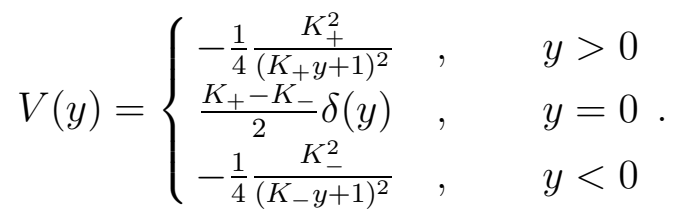

The solution $f_{0}(y)$ to the Schrödinger equation satisfying the boundary condition $\tilde{f}_{0}^{\prime}\left(0^{+}\right)-\tilde{f}_{0}^{\prime}\left(0^{-}\right)=\frac{K_{+}-K_{-}}{2} \tilde{f}_{0}(0)$ is $\tilde{f}_{0}(y) \sim\left(K_{+} y+1\right)^{1 / 2}$ for $y>0$ and $\sim\left(K_{-} y+1\right)^{1 / 2}$ for $y<0$. So, as in the dilatonic domain wall case, the KK mode zero mode is constant: $f_{0}(y)=e^{-2 A} \tilde{f}_{0}(y)=$ constant. The normalized form of the zero mode is $f_{0}(y)=N_{0}=\sqrt{\frac{3 K_{+} K_{-}}{2\left(K_{+}-K_{-}\right)}}$. Similarly as in the dilatonic domain wall case, one obtains the action for the massless dilaton $\varphi_{0}$ in the four-dimensional flat spacetime by substituting the zero mode field $\Phi=\varphi_{0} f_{0}$ into the bulk action (31). In this case, the integration interval for the extra spatial coordinate is $-1 / K_{-} \leq y \leq-1 / K_{+}$.

\subsection{Abelian gauge field}

The action for the bulk Abelian gauge field $A_{M}\left(x^{\mu}, y\right)$ is

$$
S_{\text {bulk }} \supset \int d x^{4} d y \sqrt{-G}\left[-\frac{1}{4} G^{M N} G^{R S} F_{M R} F_{N S}+\frac{1}{2} m^{2} G^{M N} A_{M} A_{N}\right],
$$

from which we obtain the following equation of motion for $A_{M}$ :

$$
\frac{1}{\sqrt{-G}} \partial_{M}\left[\sqrt{-G} G^{M N} G^{R S} F_{N S}\right]+m^{2} G^{R S} A_{S}=0
$$

By taking the divergence (defined as $\nabla_{M} V^{M} \equiv \frac{1}{\sqrt{-G}} \partial_{M}\left(\sqrt{-G} V^{M}\right)$ ) of this equation, one has $m^{2} \nabla_{M} A^{M}=0$. For $m \neq 0$, one obtains the gauge condition $\nabla_{M} A^{M}=0$ on a massive $A_{M}$. By using this gauge condition, one can eliminate one of the five components of $A_{M}$, which we choose as $A_{y}$. Then, the gauge condition $\nabla_{M} A^{M}=0$ along with the gauge choice $A_{y}=0$ and the five-dimensional metric $G_{M N}$ of the form (4) implies $\eta^{\mu \nu} \partial_{\mu} A_{\nu}=0$. In the case of massless $(m=0)$ bulk Abelian gauge field $A_{M}$, one can also choose the gauge $A_{y}=0=\eta^{\mu \nu} \partial_{\mu} A_{\nu}$ by using the gauge degrees of freedom. In the $A_{y}=0=\eta^{\mu \nu} \partial_{\mu} A_{\nu}$ gauge, the equation of motion (43) for $A_{M}$ takes the following form:

$$
\left[\eta^{\mu \nu} \partial_{\mu} \partial_{\nu}+\partial_{y} e^{2 A} \partial_{y}+m^{2} e^{2 A}\right] A_{\rho}=0 .
$$

To consider the KK mode of the bulk Abelian gauge field $A_{\rho}$ with mass $m_{n}$, only, we decompose $A_{\rho}$ as

$$
A_{\rho}\left(x^{\mu}, y\right)=a_{\rho}^{(n)}\left(x^{\mu}\right) f_{n}(y)
$$

\footnotetext{
${ }^{4}$ We define $A^{\prime}(y)$ at $y=0$ as $A^{\prime}(0) \equiv \lim _{\varepsilon \rightarrow 0^{+}} \frac{A(\varepsilon)-A(-\varepsilon)}{\varepsilon-(-\varepsilon)}$, and similarly for $A^{\prime \prime}(0)$. And we used the fact that $a+b \delta(y)=b \delta(y)$ at $y=0$ for any finite constant $a$.
} 
and require $a_{\rho}^{(n)}$ to satisfy the following Proca equation for an Abelian gauge field with mass $m_{n}$ in the Lorentz gauge in flat four-dimensional spacetime:

$$
\left[\eta^{\mu \nu} \partial_{\mu} \partial_{\nu}+m_{n}^{2}\right] a_{\rho}^{(n)}=0
$$

Then, the equation of motion (44) reduces to the following Sturm-Liouville equation:

$$
\partial_{y}\left[e^{2 A} \partial_{y} f_{n}\right]+m^{2} e^{2 A} f_{n}=m_{n}^{2} f_{n}
$$

So, the KK modes with different masses are orthogonal to each other with respect to the weighting function $w(y)=1$, provided the boundary condition $\left.f_{m}^{*} e^{2 A} f_{n}^{\prime}\right|_{y=b} ^{y=b}=0$ is satisfied.

By using a new $y$-dependent function $\tilde{f}_{n}=e^{A} f_{n}$, one can bring Eq. (47) to the following Schrödinger equation form:

$$
-\frac{d^{2} \tilde{f}_{n}}{d y^{2}}+V(y) \tilde{f}_{n}=m^{2} \tilde{f}_{n}
$$

with the potential

$$
V(y)=A^{\prime \prime}+\left(A^{\prime}\right)^{2}+m_{n}^{2} e^{-2 A} .
$$

From now on, we consider only the zero mode $\left(m_{0}=0\right)$ of the massless bulk Abelian gauge field $(m=0)$.

First, for the dilatonic domain wall solution (24), the potential (49) in the Schrödinger equation (48) takes the following form:

$$
V(y)=\frac{4 K^{2}}{81 a^{4}} \frac{4-9 a^{2}}{(1-K|y|)^{2}}-\frac{8 K}{9 a^{2}} \delta(y) .
$$

The solution to the Schrödinger equation that satisfies the boundary condition $\tilde{f}_{0}^{\prime}\left(0^{+}\right)-$ $\tilde{f}_{0}^{\prime}\left(0^{-}\right)=-\frac{8 K}{9 a^{2}} \tilde{f}_{0}(0)$ is $\tilde{f}_{0}(y) \sim(1-K|y|)^{\frac{4}{9 a^{2}}}$. So, the KK zero mode is constant: $f_{0}(y)=$ $e^{-A} \tilde{f}_{0}=$ constant. The normalized form of the zero mode is $f_{0}(y)=N_{0}=\sqrt{K / 2}$. We obtain the following effective action for the massless Abelian gauge field $a_{\mu}^{(0)}$ (with the field strength $\left.f_{\mu \nu}^{(0)}=\partial_{\mu} a_{\nu}^{(0)}-\partial_{\nu} a_{\mu}^{(0)}\right)$ in the four-dimensional flat spacetime by substituting the zero mode field $A_{\mu}=a_{\mu}^{(0)} f_{0}$ into the action (42) for the bulk massless Abelian gauge field $(m=0)$ :

$$
\begin{aligned}
-\frac{1}{4} \int d x^{4} d y \sqrt{-G} G^{M N} G^{R S} F_{M R} F_{N S} & =-\frac{1}{4} \int_{-1 / K}^{1 / K} d y f_{0}^{2} \int d x^{4} \eta^{\mu \nu} \eta^{\rho \sigma} f_{\mu \rho}^{(0)} f_{\nu \sigma}^{(0)} \\
& =-\frac{1}{4} \int d x^{4} \eta^{\mu \nu} \eta^{\rho \sigma} f_{\mu \rho}^{(0)} f_{\nu \sigma}^{(0)}
\end{aligned}
$$


Second, for the self-tuning flat domain wall solution (26), the potential is given by

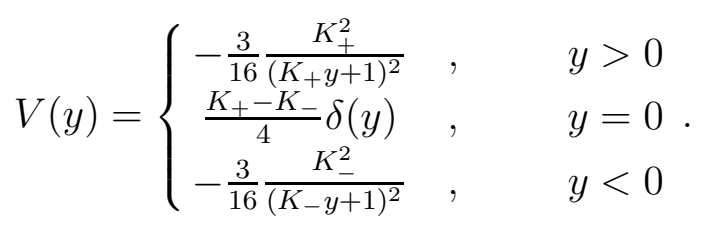

The solution $\tilde{f}_{0}(y)$ to the Schrödinger equation satisfying the boundary condition $\tilde{f}_{0}^{\prime}\left(0^{+}\right)-\tilde{f}_{0}^{\prime}\left(0^{-}\right)=\frac{K_{+}-K_{-}}{4} \tilde{f}_{0}(0)$ is $\tilde{f}_{0}(y) \sim\left(1+K_{+} y\right)^{1 / 4}$ for $y>0$ and $\sim\left(1+K_{-} y\right)^{1 / 4}$ for $y<0$. So, as in the dilatonic domain wall case, the KK zero mode is $y$-independent: $f_{0}(y)=e^{-A} \tilde{f}_{0}=$ constant. The normalized form of the KK zero mode is $f_{0}(y)=N_{0}=$ $\sqrt{K_{+} K_{-} /\left(K_{+}-K_{-}\right)}$. Similarly as in the case of the dilatonic domain wall, we obtain the four-dimensional effective action for the massless Abelian gauge field $a_{\mu}^{(0)}$ in flat spacetime by plugging the zero mode field $A_{\mu}=a_{\mu}^{(0)} f_{0}$ into the bulk action.

Note, in the case of the non-dilatonic domain wall of the original RS model $[3,4,5]$, the KK zero mode $f_{0}=$ constant is not normalizable: $\int_{-\infty}^{\infty} d y f_{0}^{2}=\infty$ for the warp factor $e^{2 A}=e^{-2 k|y|}$ which is defined on $-\infty<y<\infty$. So, the four-dimensional effective action for the massless Abelian gauge field cannot be obtained, unless one restricts the allowed values of $y$, for example, by regarding the extra dimension to be a segment $S^{1} / \mathbf{Z}_{2}$ as in the first RS model [3].

\subsection{Spinor field}

The action for the bulk spin- $1 / 2$ fermion $\Psi\left(x^{\mu}, y\right)$ is ${ }^{5}$

$$
S_{\text {bulk }} \supset \int d x^{4} d y \sqrt{G} i \bar{\Psi} \Gamma^{M} D_{M} \Psi
$$

where $D_{M} \equiv \partial_{M}+\frac{1}{4} \omega_{M A B} \gamma^{A B}$ is the gravitational covariant derivative on a spinor. Here, $\omega_{M A B}$ is the usual spacetime spin-connection. The convention for the spacetime vector indices are $M, N, P[A, B, C]$ for the five-dimensional curved [flat-tangent] spacetime indices, $\mu, \nu, \rho[\alpha, \beta, \gamma]$ for the four-dimensional curved [flat-tangent] spacetime indices, and $y$ and 5 respectively for the curved and flat extra spatial indices. The flat space gamma matrices $\gamma^{A}$ satisfying $\left\{\gamma^{A}, \gamma^{B}\right\}=2 \eta^{A B}$ are give by $\gamma^{A}=\left(\gamma^{\alpha}, i \gamma^{5}\right)$. The curved space gamma matrices $\Gamma^{M} \equiv E_{A}^{M} \gamma^{A}$ satisfy $\left\{\Gamma^{M}, \Gamma^{N}\right\}=2 G^{M N}$, where $E_{A}^{M}$ is the inverse of the Fünfbein $E_{M}^{A}$.

From the above action, we obtain the following equation of motion for the bulk spinor $\Psi$ :

$$
i\left(\Gamma^{M} \partial_{M}+\frac{1}{4} \Gamma^{M} \omega_{M A B} \gamma^{A B}\right) \Psi=0,
$$

\footnotetext{
${ }^{5}$ Note, for bulk fermions, we use the mostly negative metric signature convention, i.e., $\eta_{\mu \nu}=$ $\operatorname{diag}(1,-1,-1,-1,-1)$.
} 
which reduces to the following form after the metric (4) is substituted:

$$
i\left(e^{-A} \gamma^{\alpha} \partial_{\alpha}+i \gamma^{5} \partial_{y}+2 i \partial_{y} A \gamma^{5}\right) \Psi=0
$$

To consider the KK mode with mass $m_{n}$, only, we decompose the bulk spinor $\Psi=$ $\Psi^{R}+\Psi^{L}$ as $\Psi^{R, L}\left(x^{\mu}, y\right)=\psi_{n}^{R, L}\left(x^{\mu}\right) f_{n}^{R, L}(y)$ and require $\psi_{n}=\psi_{n}^{R}+\psi_{n}^{L}$ to satisfy the following Dirac equation for a spinor with mass $m_{n}$ in flat four-dimensional spacetime:

$$
\left[i \gamma^{\alpha} \partial_{\alpha}-m_{n}\right] \psi_{n}=0
$$

Here, $\Psi^{R, L} \equiv \frac{1}{2}\left(1 \pm \gamma^{5}\right) \Psi$ and similarly for $\psi_{n}^{R, L}$. Then, the equation of motion (55) reduces to the following form:

$$
\left(\partial_{y}+2 \partial_{y} A\right) f_{n}^{R, L}= \pm m_{n} e^{-A} f_{n}^{L, R} .
$$

In the following, we study the KK zero mode $\left(m_{0}=0\right)$.

First, for the dilatonic domain wall solution (24), the KK zero mode is

$$
f_{0}(y) \sim(1-K|y|)^{-\frac{8}{9 a^{2}}}
$$

We check the normalizability of the KK zero mode by plugging the zero mode field $\Psi=\psi_{0}\left(x^{\mu}\right) f_{0}(y)$ into the bulk action:

$$
\int d x^{4} d y \sqrt{G} i \bar{\Psi} \Gamma^{M} D_{M} \Psi=\int_{-1 / K}^{1 / K} d y e^{3 A} f_{0}^{2} \int d x^{4} i \bar{\psi}_{0} \gamma^{\alpha} \partial_{\alpha} \psi_{0}
$$

We see that the KK zero mode is normalizable, provided $a^{2}>4 / 9$. The KK zero mode including the normalization factor is $f_{0}(y)=\sqrt{\left(\frac{1}{2}-\frac{2}{9 a^{2}}\right) K}(1-K|y|)^{-\frac{8}{9 a^{2}}}$.

Second, for the self-tuning flat domain wall solution (26), the KK zero mode is

$$
f_{0}(y) \sim\left\{\begin{array}{ll}
\left(K_{+} y+1\right)^{-\frac{1}{2}}, & y>0 \\
\left(K_{-} y+1\right)^{-\frac{1}{2}}, & y<0
\end{array} .\right.
$$

One can show that this KK zero mode is normalizable by plugging the zero mode field $\Psi=\psi_{0}\left(x^{\mu}\right) f_{0}(y)$ into the bulk action, as we did for the dilatonic domain wall case. The normalization factor for the KK zero mode in this case is $N_{0}=\sqrt{\frac{3 K_{+} K_{-}}{4\left(K_{+}-K_{-}\right)}}$.

For the non-dilatonic RS domain wall, the zero mode $f_{0}(y) \sim e^{2 k|y|}$, is not normalizable, i.e., $\int_{-\infty}^{\infty} d y e^{3 A} f_{0}^{2} \sim \int_{-\infty}^{\infty} d y e^{k|y|}=\infty$, unless one restricts the allowed values of $y$ within a finite interval. 


\subsection{Gravitino}

The action for the bulk gravitino $\Psi_{M}$ is

$$
S_{\text {bulk }} \supset \int d^{4} x d y \sqrt{-G} \frac{1}{2} \bar{\Psi}_{M} \Gamma^{M N P} D_{N} \Psi_{P} .
$$

So, the equation of motion for the gravitino is

$$
\Gamma^{M N P} D_{N} \Psi_{P}=0 .
$$

We choose the gauge $\Psi_{y}=0$.

To consider the KK zero mode of $\Psi_{\mu}$, we decompose it as

$$
\Psi_{\mu}\left(x^{\nu}, y\right)=\psi_{\mu}^{(0)}\left(x^{\nu}\right) f_{0}(y),
$$

and require $\psi_{\mu}^{(0)}$ to satisfy the following Rarita-Schwinger equation for the massless gravitino in flat four-dimensional spacetime:

$$
\gamma^{\alpha \beta \delta} \partial_{\beta} \psi_{\delta}^{(0)}=0,
$$

along with the gauge conditions $\partial^{\alpha} \psi_{\alpha}^{(0)}=0=\gamma^{\alpha} \psi_{\alpha}^{(0)}$. Then, the equation of motion (62) takes the following form:

$$
\left[\partial_{y}+\partial_{y} A\right] f_{0}=0
$$

First, for the dilatonic domain wall solution (24), the KK zero mode is

$$
f_{0}(y) \sim(1-K|y|)^{-\frac{4}{9 a^{2}}} .
$$

To check the normalizability of the KK zero mode, we substitute the zero mode field $\Psi_{\mu}=\psi_{\mu}^{(0)} f_{0}$ into the bulk action:

$$
\int d^{4} x d y \sqrt{-G} \frac{1}{2} \bar{\Psi}_{M} \Gamma^{M N P} D_{N} \Psi_{P}=\int_{-1 / K}^{1 / K} d y e^{A} f_{0}^{2} \int d x^{4} \frac{1}{2} \bar{\psi}_{\alpha}^{(0)} \gamma^{\alpha \beta \delta} \partial_{\beta} \psi_{\delta}^{(0)} .
$$

We see that the KK zero mode (66) is normalizable and its normalization factor is $N_{0}=\sqrt{K / 2}$.

Second, for the self-tuning flat domain wall solution (26), the KK zero mode is

$$
f_{0}(y) \sim\left\{\begin{array}{ll}
\left(K_{+} y+1\right)^{-\frac{1}{4}}, & y>0 \\
\left(K_{-} y+1\right)^{-\frac{1}{4}}, & y<0
\end{array} .\right.
$$

Similarly as in the dilatonic domain wall case, one can show that this KK zero mode is normalizable. The normalization factor of the KK zero mode $f_{0}(y)$, in this case, is $N_{0}=\sqrt{K_{+} K_{-} /\left(K_{+}-K_{-}\right)}$.

For the non-dilatonic RS domain wall, the zero mode $f_{0}(y) \sim e^{k|y|}$ is not normalizable, i.e., $\int_{-\infty}^{\infty} d y e^{A} f_{0}^{2} \sim \int_{-\infty}^{\infty} d y e^{k|y|}=\infty$, unless one restricts the allowed values of $y$ within a finite interval. 


\subsection{Graviton}

In our previous works, we observed [6] that the RS type scenario can be extended to the extreme dilatonic domain walls and showed [7] that indeed the dilatonic domain walls effectively compactify gravity to one lower dimensions by calculating the graviton KK zero mode effective action. Later, this was further confirmed [25] by explicitly constructing the normalizable graviton KK zero modes. Although the explicit graviton KK zero modes are given in Ref. [25], we shall calculate them again since we are using different parametrization of solution, which we find to be more convenient.

For the purpose of studying the KK zero mode of graviton, it is more convenient to consider the domain wall metric in conformally flat form. (The reason is that in such coordinate frame the metric perturbation in the RS gauge around the domain wall metric takes the form of the Schrödinger equation (76) in the below.) This is achieved by transforming the extra spatial coordinate to new one, which we denote as $z$. The conformal factor for the domain wall metric and the dilaton are ${ }^{6}$

$$
\mathcal{C}(z)=(1+\bar{K}|z|)^{\frac{8}{9 a^{2}-4}}, \quad \phi=\frac{9 a}{9 a^{2}-4} \ln (1+\bar{K}|z|)+C,
$$

for the dilatonic domain wall, and

$$
\begin{aligned}
\mathcal{C}(z) & = \begin{cases}\left(1+\bar{K}_{+} z\right)^{\frac{2}{3}}, & z>0 \\
\left(1+\bar{K}_{-} z\right)^{\frac{2}{3}}, & z<0\end{cases} \\
\phi & = \begin{cases}\eta_{+} \ln \left(1+\bar{K}_{+} z\right)+C, & y>0 \\
\eta_{-} \ln \left(1+\bar{K}_{-} z\right)+C, & y<0\end{cases}
\end{aligned}
$$

for the self-tuning flat domain wall, where $\bar{K}$ and $\bar{K}_{ \pm}$are defined as

$$
\bar{K} \equiv \frac{4-9 a^{2}}{6} e^{-a C} \sqrt{\frac{3}{16-9 a^{2}} \Lambda}, \quad \bar{K}_{ \pm} \equiv \frac{3}{4} K_{ \pm} .
$$

To study the KK modes of graviton in the bulk background of the domain walls, we consider the following small fluctuation around the domain wall metric:

$$
G_{M N} d x^{M} d x^{N}=\mathcal{C}(z)\left[\left(\eta_{\mu \nu}+h_{\mu \nu}\right) d x^{\mu} d x^{\nu}+d z^{2}\right]
$$

where metric perturbation $h_{\mu \nu}\left(x^{\rho}, z\right)$ is assumed to satisfy the transverse traceless gauge condition $h^{\mu}{ }_{\mu}=0=\partial^{\mu} h_{\mu \nu}$. The $(\mu, \nu)$-component of the Einstein equations is approximated, to the first order in $h_{\mu \nu}$, to

$$
\left[\square_{x}+\partial_{z}^{2}+\frac{3}{2} \frac{\partial_{z} \mathcal{C}}{\mathcal{C}} \partial_{z}\right] h_{\mu \nu}=0
$$

\footnotetext{
${ }^{6}$ Making use of the invariance of conformally flat form of the domain wall metric Ansatz under the translation in $z$, we choose the coordinate such that $y=0$ corresponds to $z=0$. Then, the requirement $\mathcal{C}(z=0)=e^{2 A(y=0)}=1$ fixes the conformal factor to take the following forms.
} 
where $\square_{x} \equiv \eta^{\mu \nu} \partial_{\mu} \partial_{\nu}$. To consider the KK mode with mass $m_{n}$, only, we decompose $h_{\mu \nu}$ as $h_{\mu \nu}\left(x^{\rho}, z\right)=\hat{h}_{\mu \nu}^{(n)}\left(x^{\rho}\right) f_{n}(z)$ and require $\hat{h}_{\mu \nu}^{(n)}$ to satisfy $\square_{x} \hat{h}_{\mu \nu}^{(n)}=m_{n}^{2} \hat{h}_{\mu \nu}^{(n)}$. Then, the linearized Einstein equation (73) reduces to the following form:

$$
\left[\partial_{z}^{2}+\frac{3}{2} \frac{\partial_{z} \mathcal{C}}{\mathcal{C}} \partial_{z}+m_{n}^{2}\right] f_{n}=0 .
$$

Had we used $y$ as the extra spatial coordinate, the equation (74) satisfied by the KK mode $f_{n}$ with mass $m_{n}$ would have taken the following form of the Sturm-Liouville equation:

$$
\partial_{y}\left[e^{4 A} \partial_{y} f_{n}\right]+m_{n}^{2} e^{2 A} f_{n}=0,
$$

from which we know that the KK modes are orthogonalized with the respect to the weighting function $w(y)=e^{2 A}$, provided the boundary condition $\left.f_{m}^{*} e^{4 A} f_{n}^{\prime}\right|_{y=a} ^{y=b}=0$ is satisfied, or with respect to the weighting function $w(z)=\mathcal{C}^{3 / 2}$ if $z$ is used as the extra spatial coordinate.

In terms of a new $z$-dependent function defined as $\tilde{f}_{n} \equiv \mathcal{C}^{3 / 4} f_{n}$, Eq. (74) takes the following form of the Schrödinger equation:

$$
-\frac{d^{2} \tilde{f}_{n}}{d z^{2}}+V(z) \tilde{f}_{n}=m_{n}^{2} \tilde{f}_{n}
$$

with the potential

$$
V(z)=\frac{3}{16}\left[4 \frac{\mathcal{C}^{\prime \prime}}{\mathcal{C}}-\left(\frac{\mathcal{C}^{\prime}}{\mathcal{C}}\right)^{2}\right] .
$$

In the following, we study the KK zero mode $\left(m_{0}=0\right)$, for which $\hat{h}_{\mu \nu}^{(0)}$ satisfies the linearized vacuum Einstein equation $\square_{x} \hat{h}_{\mu \nu}^{(0)}=0$ in the Lorentz gauge.

First, for the dilatonic domain wall solution (69), the potential (77) in the Schrödinger equation (76) takes the following form:

$$
V(z)=\frac{6 \bar{K}^{2}}{\left(9 a^{2}-4\right)^{2}} \frac{10-9 a^{2}}{(1+\bar{K}|z|)^{2}}-\frac{12 \bar{K}}{4-9 a^{2}} \delta(z) .
$$

Note, the $\delta$-function potential is always attractive, implying that the KK zero mode solution can always be supported. The solution $\tilde{f}_{0}$ to the Schrödinger equation satisfying the boundary condition $\tilde{f}_{0}^{\prime}\left(0^{+}\right)-\tilde{f}_{0}^{\prime}\left(0^{-}\right)=-\frac{12 \bar{K}}{4-9 a^{2}} \tilde{f}_{0}(0)$ is $\tilde{f}_{0} \sim(1+\bar{K}|z|)^{-\frac{6}{4-9 a^{2}}}$. So, the KK zero mode is $z$-independent: $f_{0}(z)=\mathcal{C}^{-3 / 4} \tilde{f}_{0}=$ constant. The normalization factor for the KK zero mode is $N_{0}=\sqrt{\left(\frac{1}{2}+\frac{4}{9 a^{2}}\right) K}$.

Second, for the self-tuning flat domain wall solution (70), the potential has the following form:

$$
V(z)=\left\{\begin{array}{cll}
-\frac{1}{4} \frac{\bar{K}_{+}^{2}}{\left(K_{+} z+1\right)^{2}} & , & z>0 \\
\frac{1}{2}\left(\bar{K}_{+}-\bar{K}_{-}\right) \delta(z) & , & z=0 . \\
-\frac{1}{4} \frac{\bar{K}_{-}^{2}}{\left(K_{-} z+1\right)^{2}} & , & z<0
\end{array}\right.
$$


As in the dilatonic domain wall case, the $\delta$-function potential is always attractive. The solution $\tilde{f}_{0}(z)$ to the Schrödinger equation satisfying the boundary condition $\tilde{f}_{0}^{\prime}\left(0^{+}\right)-$ $\tilde{f}_{0}^{\prime}\left(0^{-}\right)=\frac{1}{2}\left(\bar{K}_{+}-\bar{K}_{-}\right) \tilde{f}_{0}(0)$ is $\tilde{f}_{0}(z) \sim\left(\bar{K}_{+} z+1\right)^{1 / 2}$ for $z>0$ and $\sim\left(\bar{K}_{-} z+1\right)^{1 / 2}$ for $z<0$. So, as in the dilatonic domain wall case, the KK zero mode is $z$-independent: $f_{0}(z)=\mathcal{C}^{-3 / 4} \tilde{f}_{0}=$ constant. The normalization constant for the KK zero mode $f_{0}$ is $N_{0}=\sqrt{\frac{3}{2} \frac{K_{+} K_{-}}{K_{+}-K_{-}}}$.

In the following, we obtain the KK zero mode effective actions for the graviton in the bulk backgrounds of the dilatonic and the self-tuning domain walls. Since we have shown that the KK zero mode $h_{\mu \nu}=\hat{h}_{\mu \nu}^{(0)} f_{0}$ of the graviton is independent of the extra spatial coordinate $z$, we consider the following form of the bulk metric:

$$
G_{M N} d x^{M} d x^{N}=\mathcal{C}(z)\left[g_{\mu \nu}\left(x^{\rho}\right) d x^{\mu} d x^{\nu}+d z^{2}\right],
$$

where the conformal factor $\mathcal{C}$ is given by Eq. (69) or (70). The dilaton $\phi(z)$ is given by Eq. (69) or (70). A useful formula for obtaining the KK zero mode effective actions is

$$
\sqrt{-G} \mathcal{R}_{G}=\sqrt{-g} \mathcal{C}^{\frac{3}{2}}\left[\mathcal{R}_{g}-4 \frac{\mathcal{C}^{\prime \prime}}{\mathcal{C}}+\left(\frac{\mathcal{C}^{\prime}}{\mathcal{C}}\right)^{2}\right] .
$$

Here, $\mathcal{R}_{G}$ and $\mathcal{R}_{g}$ are respectively the Ricci scalars for the metrics $G_{M N}$ and $g_{\mu \nu}$. If we instead use the KK zero mode bulk metric of the following form:

$$
G_{M N} d x^{M} d x^{N}=e^{2 A(y)} g_{\mu \nu}\left(x^{\rho}\right) d x^{\mu} d x^{\nu}+d y^{2},
$$

then the corresponding formula would be

$$
\sqrt{-G} \mathcal{R}_{G}=\sqrt{-g}\left[e^{2 A} \mathcal{R}_{g}-4 e^{4 A}\left\{2 A^{\prime \prime}+5\left(A^{\prime}\right)^{2}\right\}\right],
$$

but the resulting expression for the effective actions will be the same.

First, we consider the effective action in the bulk of the dilatonic domain wall. In our previous work [7], we obtained the effective action for the case where $K$ defined in Eq. (6) is positive [negative] in the region $y<0[y>0]$ for the $\Delta<0$ case, only. In this case, the tension $\sigma_{\mathrm{DW}}$ of the wall takes the positive value given by Eq. (7) with $D=5$. In this paper, we shall assume that $K$ defined in Eq. (6) is positive [negative] in the region $y<0[y>0]$ even for the $\Delta>0$ case, as well as for the $\Delta<0$ case. Substituting the Ansätze ${ }^{7}$ for the fields given by Eqs. (69) and (80) into the total action, we obtain the following:

$$
S=\frac{1}{2 \kappa_{5}^{2}} \int d^{5} x \sqrt{-G}\left[\mathcal{R}_{G}-\frac{4}{3} \partial_{M} \phi \partial^{M} \phi+e^{-2 a \phi} \Lambda\right]-\sigma_{D W} \int d^{4} x \sqrt{-\gamma} e^{-a \phi}
$$

\footnotetext{
${ }^{7}$ Such Ansätze are consistent with the five-dimensional equations of motion as long as the zero mode metric $g_{\mu \nu}\left(x^{\rho}\right)$ satisfies the Ricci flat condition. The Ricci flat condition is equivalent to the condition that the zero mode $g_{\mu \nu}\left(x^{\rho}\right)$ satisfies the four-dimensional Einstein equations with vanishing cosmological constant.
} 


$$
\begin{gathered}
=\frac{1}{2 \kappa_{5}^{2}} \int d^{4} x d z \sqrt{-g} \varpi \frac{12}{9 a^{2}-4}\left[\mathcal{R}_{g}-\frac{20\left(16-9 a^{2}\right) \bar{K}^{2}}{\left(4-9 a^{2}\right)^{2}} \varpi^{-2}+\frac{64 \bar{K}}{4-9 a^{2}} \delta(z)\right. \\
\left.+e^{-2 a C} \Lambda \varpi^{-2}\right]-\int d^{4} x \sqrt{-g} e^{-a C} \sigma_{\mathrm{DW}},
\end{gathered}
$$

where from Eqs. (71) and (25) we see that the cosmological constant and the tension of the wall can be expressed as

$$
\Lambda=\frac{12\left(16-9 a^{2}\right)}{\left(4-9 a^{2}\right)^{2}} e^{2 a C} \bar{K}^{2}, \quad \sigma_{\mathrm{DW}}=\frac{1}{\kappa_{5}^{2}} \frac{24}{4-9 a^{2}} e^{a C} \bar{K},
$$

and $\varpi \equiv 1+\bar{K}|z|$. After the integration over $z$ (with the integration interval $-\infty<$ $z<\infty$ for $a^{2}<4 / 9$ and $\bar{K}^{-1}<z<-\bar{K}^{-1}$ for $4 / 9<a^{2}<16 / 9$ ), we see that all the extra terms cancel out and we are left with the term for the four-dimensional general relativity (with vanishing cosmological constant) with the gravitational constant given by

$$
\kappa_{4}^{2}=\frac{8+9 a^{2}}{2\left(4-9 a^{2}\right)} \bar{K} \kappa_{5}^{2}=e^{-a C} \frac{8+9 a^{2}}{12} \sqrt{\frac{3}{16-9 a^{2}} \Lambda} \kappa_{5}^{2} .
$$

A troublesome case is the $a^{2}>16 / 9$ case (with the integration interval $\bar{K}^{-1}<z<$ $-\bar{K}^{-1}$ ), in which the integration on the $\varpi^{-2}$ terms in the action (84) diverges, whereas the effective four-dimensional gravitational constant $\kappa_{4}^{2}$ remains to have the nonzero value given by Eq. (86). (This is the case which normally would have been discarded because of the positive cosmological constant (negative $\Lambda$ in our convention).) On the other hand, the graviton KK zero mode $g_{\mu \nu}\left(x^{\rho}\right)$ in the dilatonic domain wall background (69) satisfies the four-dimensional vacuum Einstein's equation with vanishing cosmological constant term. So, it seems to be contradictory that we don't reproduce the action for the four-dimensional general relativity with vanishing cosmological constant by integrating the action (84) with respect to the extra spatial coordinate $z$. One can avoid infinity in the effective action by truncating the extra spatial dimension through the introduction of another domain wall in the region between $z=0$ and $|z|=-\bar{K}^{-1}$. This seems to be reasonable because $|z|=-\bar{K}^{-1}$ (or $|y|=K^{-1}$ ) corresponds to the curvature singularity, which has to be avoided in the brane world scenario unless there is reasonable physical significance associated with the singularity. Then, the total action has the following form:

$$
\begin{aligned}
S= & \frac{1}{2 \kappa_{5}^{2}} \int d^{4} x \int_{-z_{0}}^{z_{0}} d z \sqrt{-G}\left[\mathcal{R}_{G}-\frac{4}{3} \partial_{M} \phi \partial^{M} \phi+e^{-2 a \phi} \Lambda\right] \\
& -\sigma_{h} \int_{z=0} d^{4} x \sqrt{-\gamma^{h}} e^{-a \phi_{h}}-\sigma_{v} \int_{z=z_{0}} d^{4} x \sqrt{-\gamma^{v}} e^{-a \phi_{v}}
\end{aligned}
$$

where

$$
\sigma_{h}=-\sigma_{v}=\frac{4}{\kappa_{5}^{2}} \sqrt{\frac{3}{16-9 a^{2}} \Lambda}
$$




$$
\begin{aligned}
\gamma_{\mu \nu}^{h} & =g_{\mu \nu}, & \gamma_{\mu \nu}^{v} & =\left(1+\bar{K} z_{0}\right)^{\frac{8}{9 a^{2}-4}} g_{\mu \nu}, \\
\phi_{h} & =C, & \phi_{v} & =\frac{9 a}{9 a^{2}-4} \ln \left(1+\bar{K} z_{0}\right)+C .
\end{aligned}
$$

Going through the similar calculation as in Eq. (84), except that there will be an additional $\delta$-function term at $|z|=z_{0}$ in the integrand of the bulk action, one will find after the $z$-integration that all the extra terms are cancelled and one is left only with the curvature term. The effective four-dimensional gravitational constant in this case is given by

$$
\begin{aligned}
\kappa_{4}^{2} & =\frac{8+9 a^{2}}{2\left(4-9 a^{2}\right)} \bar{K}\left[1-\varpi_{0}^{\frac{9 a^{2}+8}{9 a^{2}-4}}\right]^{-1} \kappa_{5}^{2} \\
& =e^{-a C} \frac{8+9 a^{2}}{12} \sqrt{\frac{3}{16-9 a^{2}} \Lambda}\left[1-\varpi_{0}^{\frac{9 a^{2}+8}{9 a^{2}-4}}\right]^{-1} \kappa_{5}^{2},
\end{aligned}
$$

where $\varpi_{0}=1+\bar{K} z_{0}$. Note, even if we introduced the additional domain wall to cure the problem of diverging effective action for the $a^{2}>16 / 9$ case, this result with the additional domain wall is valid for any values of $a$.

So, we see that gravity in the bulk of the dilatonic domain wall is effectively compactified to one lower dimensions, for any values of the dilaton coupling parameter $a$, provided the extra space is truncated through the introduction of another domain wall in the case of $a^{2}>16 / 9$. For this to happen, one has to choose the sign \pm in the expression for $K$ in Eq. (6) such that the warp factor for the domain wall metric has singularity at a finite value of $y$ on both sides of the wall and therefore the warp factor decreases on both sides of the domain wall. This choice of signs corresponds to the conformal factor of the form (69) (for the $D=5$ case) when the metric tensor is transformed to take a conformally flat form. Also, in this case, the tension of the wall takes the positive value given by Eq. (7). For other choices of sign \pm in the expression for $K$ in Eq. (6), the gravity in the bulk is not effectively compactified to the Einstein gravity with zero cosmological constant in one lower dimensions and the tension of the wall is either zero or negative.

Next, we consider the effective action in the bulk of the self-tuning flat domain wall. Substituting the Ansätze for the fields given by Eqs. (70) and (80) into the total action, we obtain the following:

$$
\begin{aligned}
S & =\frac{1}{2 \kappa_{5}^{2}} \int d^{5} x \sqrt{-G}\left[\mathcal{R}_{G}-\frac{4}{3} \partial_{M} \phi \partial^{M} \phi\right]-\int d^{4} x \sqrt{-\gamma} f(\phi) \\
& =\frac{1}{2 \kappa_{5}^{2}} \int d^{4} x d z \sqrt{-g} \varpi_{ \pm}\left[\mathcal{R}_{g}-\frac{8}{3}\left(\bar{K}_{+}-\bar{K}_{-}\right) \delta(z)\right]-\int d^{4} x \sqrt{-g} f(C),
\end{aligned}
$$

where in the second line we choose the plus [the minus] sign in $\varpi_{ \pm} \equiv 1+\bar{K}_{ \pm} z$ for the $z>0[z<0]$ region and $f(C)=\left(\bar{K}_{-}-\bar{K}_{+}\right) / \kappa_{5}^{2}$. Note, a term coming from $\mathcal{R}_{G}$ 
which would potentially have diverged after the $z$-integration is cancelled by another diverging term $-\frac{4}{3} \partial_{M} \phi \partial^{M} \phi$ and we are left only with the $\delta$-function term coming from $\mathcal{R}_{G}$. After the integration over $z$ (with the integration interval $-\bar{K}_{-}^{-1}<z<-\bar{K}_{+}^{-1}$ ), we obtain the following effective action:

$$
S=\frac{1}{2 \kappa_{5}^{2}} \int d^{4} x \sqrt{-g} \frac{\bar{K}_{+}-\bar{K}_{-}}{2 \bar{K}_{+} \bar{K}_{-}}\left[\mathcal{R}_{g}-\frac{4}{3} \bar{K}_{+} \bar{K}_{-}\right] .
$$

Unexpectedly, the four-dimensional effective action for the graviton zero mode has a cosmological constant term. This is contradictory, because the graviton KK zero mode $g_{\mu \nu}\left(x^{\rho}\right)$ in the bulk of the self-tuning flat domain wall satisfies the four-dimensional vacuum Einstein equations with vanishing cosmological constant and the effective fourdimensional gravitational constant has a nonzero value given by $\kappa_{4}^{2}=\frac{2 \bar{K}_{+} \bar{K}_{-}}{\bar{K}_{+}-\bar{K}_{-}} \kappa_{5}^{2}$. It is also inconsistent with the fact that the self-tuning flat domain wall solution has the four-dimensional Poincaré invariance, which requires zero cosmological constant in four dimensions ${ }^{8}$. Ref. [26], which appeared in the preprint archive after the present work, independently observes such unexpected nonzero cosmological constant term in the effective action and resolves the problem by introducing extra domain walls at the naked singularities ${ }^{9}$ and fine-tuning their tensions to cancel out the undesirable cosmological constant term. So, such resolution spoils nice self-tuning property of the self-tuning flat domain walls. This seems to indicate that there is some other ingredient missing or some inconsistency in the scenario of self-tuning flat domain wall necessary in reproducing four-dimensional effective theory with vanishing cosmological constant without loosing nice self-tuning property. (It seems to be important to obtain the expect form of the effective action, because anyway in the RS model we usually consider a part of the complete effective action to determine the four-dimensional effective gravitational constant.) It is important to note that the existence of a solution with the Poincaré invariance in one lower dimensions and the fact that the graviton zero mode in the bulk of such solution satisfies the four-dimensional vacuum Einstein equation with zero cosmological constant do not necessarily mean that the effective action in one lower dimensions has the right expected form. An example is the non-dilatonic domain wall with exponentially increasing warp factor. The graviton zero mode in such background satisfies the four-dimensional vacuum Einstein equations with zero cosmological constant, but the four-dimensional gravitational constant is zero if we take the extra spatial dimension to be infinite in size. Additional condition that the

\footnotetext{
${ }^{8}$ I would like to thank Prof. Kallosh for pointing out inconsistency between the existence of the domain wall solution with the four-dimensional Poincaré invariance and the non-vanishing cosmological constant in the effective action, after the first version of this paper appeared in the preprint archive.

${ }^{9}$ The author feels that the extra introduced domain walls should rather be placed between the singularities and the self-tuning flat domain wall, because the singularities of the self-tuning flat domain wall are problematic regions where the induced metric vanishes, the dilaton blows up and the spacetime curvature diverges.
} 
four-dimensional gravitational constant should be nonzero seems to be also insufficient. As we have seen the case of the dilatonic domain walls with positive cosmological constant $(\Lambda<0$ in our convention), although the four-dimensional gravitational constant is nonzero, as well as the KK zero mode graviton in the domain wall bulk satisfies the four-dimensional vacuum Einstein equations with zero cosmological constant, the effective action diverges unless additional domain wall is introduced. One possible solution to the problem might be to consider all the massive KK modes of bulk graviton to cancel out the unwanted cosmological constant, but on the other hand the graviton zero mode itself is a consistent solution to the five-dimensional equations of motion provided the zero mode $g_{\mu \nu}\left(x^{\rho}\right)$ satisfies the Ricci flat condition.

\section{References}

[1] N. Arkani-Hamed, S. Dimopoulos and G. Dvali, "The hierarchy problem and new dimensions at a millimeter," Phys. Lett. B429 (1998) 263, hep-ph/9803315.

[2] I. Antoniadis, N. Arkani-Hamed, S. Dimopoulos and G. Dvali, "New dimensions at a millimeter to a Fermi and superstrings at a TeV," Phys. Lett. B436 (1998) 257, hep$\mathrm{ph} / 9804398$.

[3] L. Randall and R. Sundrum, "A large mass hierarchy from a small extra dimension," Phys. Rev. Lett. 83 (1999) 3370, hep-ph/9905221.

[4] L. Randall and R. Sundrum, "An alternative to compactification," Phys. Rev. Lett. 83 (1999) 4690, hep-th/9906064.

[5] J. Lykken and L. Randall, "The shape of gravity," hep-th/9908076.

[6] D. Youm, "Solitons in brane worlds," hep-th/9911218.

[7] D. Youm, "A note on solitons in brane worlds," hep-th/0001166.

[8] K. Behrndt and M. Cvetič, "Supersymmetric domain wall world from $D=5$ simple gauged supergravity," hep-th/9909058.

[9] K. Skenderis and P.K. Townsend, "Gravitational stability and renormalization-group flow," Phys. Lett. B468 (1999) 46, hep-th/9909070.

[10] A. Chamblin and G.W. Gibbons, "Supergravity on the brane," hep-th/9909130.

[11] R. Kallosh, A. Linde and M. Shmakova, "Supersymmetric multiple basin attractors," JHEP 11 (1999) 010, hep-th/9910021.

[12] M. Cvetič, H. Lu and C.N. Pope, "Domain walls and massive gauged supergravity potentials," hep-th/0001002.

[13] R. Kallosh and A. Linde, "Supersymmetry and the brane world," JHEP 0002 (2000) 005, hep-th/0001071.

[14] K. Behrndt and M. Cvetič, "Anti-deSitter vacua of gauged supergravities with 8 supercharges," hep-th/0001159. 
[15] N. Arkani-Hamed, S. Dimopoulos, N. Kaloper and R. Sundrum, "A small cosmological constant from a large extra dimension," hep-th/0001197.

[16] S. Kachru, M. Schulz and E. Silverstein, "Self-tuning flat domain walls in 5d gravity and string theory," hep-th/0001206.

[17] W.D. Goldberger and M.B. Wise, "Bulk fields in the Randall-Sundrum compactification scenario," Phys. Rev. D60 (1999) 107505, hep-ph/9907218.

[18] W.D. Goldberger and M.B. Wise, "Modulus stabilization with bulk fields," Phys. Rev. Lett. 83 (1999) 4922, hep-ph/9907447.

[19] W.D. Goldberger and M.B. Wise, "Phenomenology of a stabilized modulus," hep$\mathrm{ph} / 9911457$.

[20] H. Davoudiasl, J.L. Hewett and T.G. Rizzo, "Bulk gauge fields in the Randall-Sundrum model," hep-ph/9911262.

[21] A. Pomarol, "Gauge bosons in a five-dimensional theory with localized gravity," hep$\mathrm{ph} / 9911294$.

[22] B. Bajc and G. Gabadadze, "Localization of matter and cosmological constant on a brane in anti de Sitter space," hep-th/9912232.

[23] Y. Grossman and M. Neubert, "Neutrino masses and mixings in non-factorizable geometry," hep-ph/9912408.

[24] S. Chang, J. Hisano, H. Nakano, N. Okada and M. Yamaguchi, "Bulk standard model in the Randall-Sundrum background," hep-ph/9912498.

[25] C. Gomez, B. Janssen and P. Silva, "Dilatonic Randall-Sundrum theory and renormalization group," hep-th/0002042.

[26] S. Forste, Z. Lalak, S. Lavignac and H.P. Nilles, "A comment on self-tuning and vanishing cosmological constant in the brane world," Phys. Lett. B481 (2000) 360, hepth/0002164. 